\title{
Absorbing Boundary Conditions for the Discretization Schemes of the One-Dimensional Wave Equation
}

\author{
By Laurence Halpern
}

\begin{abstract}
When computing a partial differential equation, it is often necessary to introduce artificial boundaries. Here we explain a systematic method to obtain boundary conditions for the wave equation in one dimension, fitting to the discretization scheme and stable. Moreover, we give error estimates on the reflected part.
\end{abstract}

1. Introduction. In order to avoid a huge amount of calculation, when solving numerically a partial differential equation, one often introduces artificial boundaries with boundary conditions chosen so that the problem one gets is well-posed, and its solution is "as close as possible" to that of the original problem.

These boundary conditions can be either transparent, i.e., such that the solution of the boundary value problem is exactly the solution of the initial problem, but such conditions are generally global in the time variable and so numerically useless or approximated. Or they are absorbing, when the energy in the artificial domain decreases with respect to the time $t$.

This problem is of practical interest, as for instance it arises in geophysics or plasma physics, and has been studied in many cases; see [4], [8], [12].

In this paper, we study the case of acoustic waves in a one-way propagation. The transparent condition one gets on the continuous problem is a local one. But, immediate discretization leads to an ill-posed problem. The difficulty is then to obtain stable boundary conditions fitting to the discretization scheme.

We only work on the discrete problem, taking into account that the solution is already an approximate one. The methods we use are drawn from the methods developed in the theory of singularities reflection; see [8], [9].

In the first part of this paper, we explain our method for an explicit discretization scheme. We first write a transparent boundary condition, which is global in the time variable, i.e., computation of the solution at some time uses the solution at any earlier time. With the assistance of the space-discretized problem, we then derive some stable conditions, and we point out that these are not canonical. We obtain, for each of them, error estimates for the energy of the reflected part. As far as we know, such estimates are new.

In the second part, we show how this method can be extended to any discretization scheme and give the form of boundary conditions in the case of $e^{1}$ cubic finite element scheme.

Received May 21, 1981; revised September 29, 1981.

1980 Mathematics Subject Classification. Primary 65M99, 65N99. 
Similar boundary conditions have also been obtained by B. Engquist and A. Majda [5] for the same model problem. We prove here the stability of our discrete boundary conditions. We use energy methods that could be generalized to other problems (for example, wave equations with variable coefficients, model wave equations with variable discretization step...). But on the model problem the numerical results are very similar, without any proof of the fact....

2. Preliminaries. The Continuous Problem. We consider here the solution of a scalar wave equation on the axis $\mathbf{R}$, where the velocity is taken equal to 1 :

$$
\square u=\partial^{2} u / \partial t^{2}-\partial^{2} u / \partial x^{2}=0 .
$$

The initial values (initial state and initial velocity) have a compact support in the half-line $x<0$.

The following result is well known:

Proposition (TRANSPARENT BOUNDARY CONDITION). The transparent boundary condition at $x=0$ for the continuous problem (2.1) is

$$
\partial u / \partial t+\partial u / \partial x=0
$$

This result can be interpreted as follows: we introduce the Fourier transform in time $\hat{u}$ of $u$

$$
\hat{u}(x, \omega)=\frac{1}{2 \pi} \int_{\mathbf{R}} u(x, t) e^{-i \omega t} d t .
$$

Since the initial values are zero for $t<0$, we deduce that $\hat{u}(x, \omega)$ is a right-travelling wave:

$$
\text { For all } \omega \in \mathbf{R}: \hat{u}(x, \omega)=\hat{a}(\omega) e^{-i \omega x} \text {. }
$$

Motivations for the Study. Consider now a discretization of Eq. (2.1) using a second order finite difference scheme:

$$
\square u_{j}^{n}=\frac{u_{j}^{n+1}-2 u_{j}^{n}+u_{j}^{n-1}}{\Delta t^{2}}-\frac{u_{j+1}^{n}-2 u_{j}^{n}+u_{j-1}^{n}}{\Delta x^{2}}=0,
$$

with the stability condition $\gamma=\Delta t / \Delta x \leqslant 1$.

The most natural discretization of boundary condition (2.2) is the following:

$$
\frac{u_{0}^{n+1}-u_{0}^{n-1}}{2 \Delta t}+\frac{u_{0}^{n}-u_{-1}^{n}}{\Delta x}=0
$$

The boundary condition (4) leads to an ill-posed boundary problem:

In order to prove this result, we apply the following criterion*: If the associated problem is well-posed, there is no solution of Eqs. (2.3) and (2.4) that decreases exponentially as $j$ tends to $-\infty$, and increases exponentially as $t$ tends to $+\infty$. Let

$$
v_{j}^{n}=(-1)^{n+j} e^{(k j \Delta x+\omega n \Delta t)},
$$

where

$$
e^{k \Delta x}=\frac{4-\gamma^{2}}{\gamma^{2}} ; \quad e^{\omega \Delta t}=\frac{2+\gamma}{2-\gamma} .
$$

$v_{j}^{n}$ is clearly a solution of the problem.

${ }^{*}$ Cf. Kreiss [7]. 


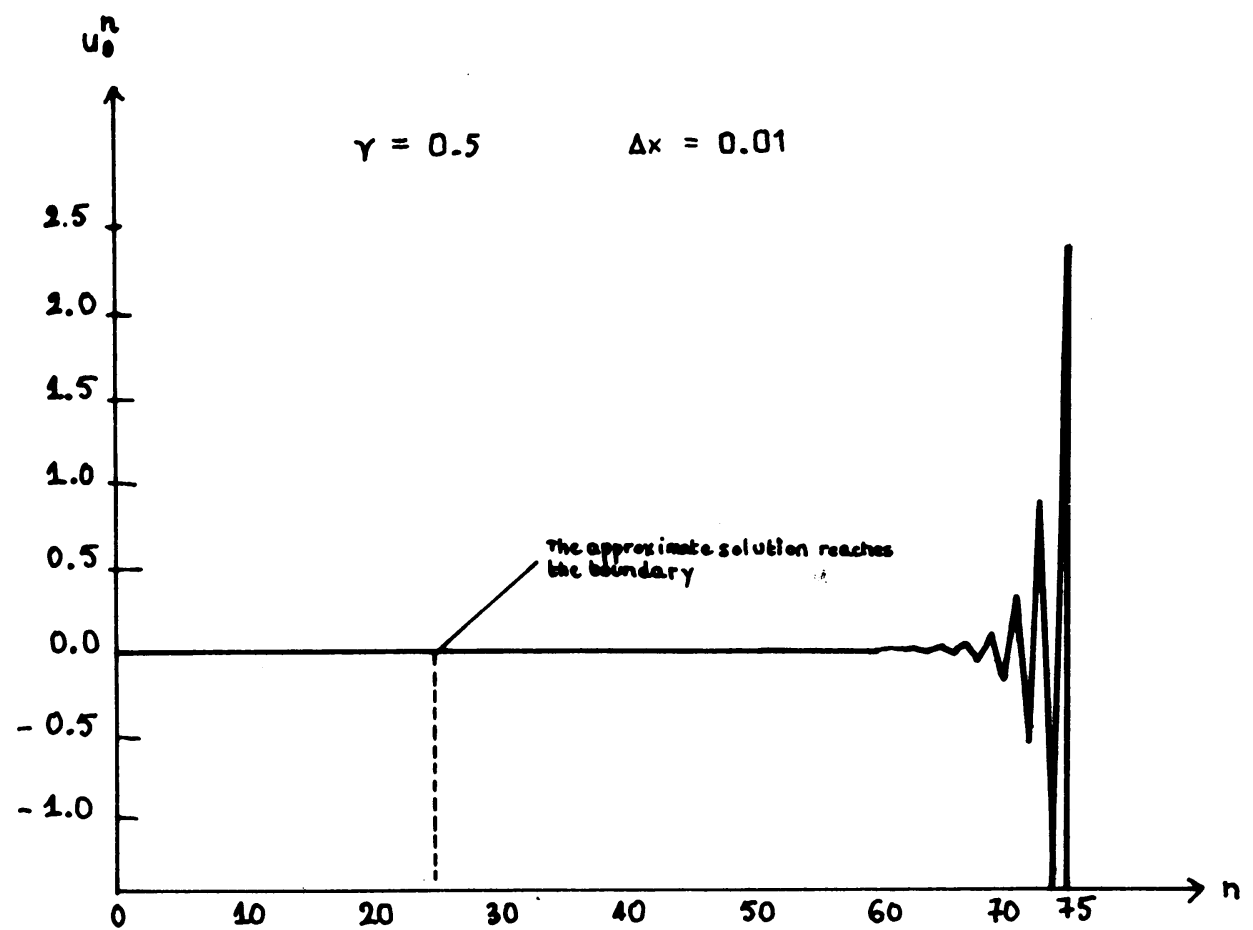

FIGURE 1

Beginning of instability: Approximate solution on the boundary in terms of time-step $n$, up to $n=75$, i.e. the time $T=75 \Delta t$

Figure 1 represents the solution of Eqs. (2.3), (2.4) with smooth initial data on the boundary $j=0$ in terms of time-steps, and points out the beginning of instability.

3. Approximate Boundary Conditions for the Second Order Finite Difference Discretization Scheme. We will here consider an explicit discretization scheme of the following form:

$$
\frac{u_{j}^{n+1}-2 u_{j}^{n}+u_{j}^{n-1}}{\Delta t^{2}}-\frac{u_{j+1}^{n}-2 u_{j}^{n}+u_{j-1}^{n}}{\Delta x^{2}}=0,
$$

where $\Delta x$ denotes the space-step, $\Delta t$ the time-step.

We assume that the stability condition for the discretization scheme is fulfilled:

$$
\gamma=\Delta t / \Delta x \leqslant 1 \text {. }
$$

First, let us introduce our notation:

$$
\begin{array}{ll}
D_{+}^{x}\left(u_{j}^{n}\right)=\frac{u_{j+1}^{n}-u_{j}^{n}}{\Delta x} ; & D_{-}^{x}\left(u_{j}^{n}\right)=\frac{u_{j}^{n}-u_{j-1}^{n}}{\Delta x} ; \quad D_{0}^{x}=\frac{D_{+}^{x}+D_{-}^{x}}{2}, \\
D_{+}^{t}\left(u_{j}^{n}\right)=\frac{u_{j}^{n+1}-u_{j}^{n}}{\Delta t} ; & D_{-}^{t}\left(u_{j}^{n}\right)=\frac{u_{j}^{n}-u_{j}^{n-1}}{\Delta t} ; \quad D_{0}^{t}=\frac{D_{+}^{t}+D_{-}^{t}}{2} .
\end{array}
$$

We introduce the exterior problem on $] 0,+\infty[$ with the boundary condition

$$
\frac{u_{0}^{n+1}-u_{0}^{n}}{\Delta t}=\varphi^{n}
$$


on $] 0,+\infty[$, the initial values are 0 , and so we can obtain a transparent boundary condition by a discrete convolution between the discrete time-derivative and the discrete space-derivative.

Proposition 3.1. The transparent boundary condition at $j=0$ is

$$
\begin{aligned}
D_{+}^{t}\left(u_{0}^{n}\right)= & \left(1-\gamma^{2}\right) D_{+}^{t}\left(u_{1}^{n-1}\right)+\gamma\left(\delta^{3} D_{+}^{t}\left(u_{1}^{n-2}\right)+\cdots+\delta^{n} D_{+}^{t}\left(u_{1}^{1}\right)\right) \\
& -\gamma D_{-}^{x}\left(u_{1}^{n}\right) .
\end{aligned}
$$

$\delta^{n}$ is the impulsional response, i.e., the solution of the exterior problem when $\varphi^{n}=\delta_{2, n}$ (Dirac function at $n=2$ ).

This transparent boundary condition is global: in order to compute the solution at the $n$-time-step, we must have information for all previous times. Therefore, we shall investigate approximate boundary conditions which are local in time and lead to a well-posed boundary problem.

In order to obtain these boundary conditions, we first introduce the semidiscretized problem with space-discretization only:

$$
\frac{d^{2} u_{j}}{d t^{2}}-\frac{u_{j+1}-2 u_{j}+u_{j-1}}{h^{2}}=0,
$$

where $h$ is the space-step.

We shall obtain for this problem an approximate boundary condition and then deduce by time-discretization the desired boundary conditions.

3.1. Boundary Conditions for the Space-Discretized Problem. We first introduce an auxiliary boundary problem on the half-axis $x>0$ and get the transparent boundary condition at point $j=0$. This is given by an integro-differential relation between $u_{-1}$ and $u_{0}$. We then deduce local boundary conditions, by approximation of the kernel, when the wavelength is large compared to $h$.

3.1.1. Transparent Boundary Conditions. We introduce the initial boundary problem on the half-axis $j \geqslant 0$

$$
\left\{\begin{array}{l}
\frac{d^{2} v_{j}}{d t^{2}}-\frac{v_{j+1}-2 v_{j}+v_{j-1}}{h^{2}}=0 ; \quad j \geqslant 0 . \\
\text { The initial values are identically zero. } \\
\text { The boundary condition is } d v_{0} / d t=D(t) .
\end{array}\right.
$$

We assume that $D$ satisfies the regularity condition

$$
D \in L^{2}(] 0,+\infty[) \text {. }
$$

The following lemma gives a result on the regularity of $v_{j}$ and the explicit form of the Fourier transform of $v_{j}$ :

LEMMA 3.1. Let $\left(v_{j}\right)_{j>0}$ be a solution of problem (1.5); under the regularity condition (1.6), we have the following estimates:

$$
\begin{gathered}
\forall j \geqslant 0 ; \quad \frac{d v_{j}}{d t} \in L^{2}(] 0,+\infty[) \text { and }\left\|\frac{d v_{j}}{d t}\right\|_{L^{2}} \leqslant\|D\|_{L^{2}} \\
\forall j \geqslant 0 ; \quad \frac{v_{j+1}-v_{j}}{h} \in L^{2}(] 0,+\infty[) \text { and }\left\|\frac{v_{j+1}-v_{j}}{h}\right\|_{L^{2}} \leqslant\|D\|_{L^{2}} .
\end{gathered}
$$


Moreover the Fourier transform of $d v_{j} / d t$ is given by

$$
\forall j \geqslant 0, \quad \frac{\widehat{d v}_{j}}{d t}(\omega)=\hat{D}(\omega) r_{1}^{j}(\omega h),
$$

where $r_{1}$ is the root of the characteristic equation $r^{2}-2\left(1-\omega^{2} h^{2} / 2\right) r+1=0$, given by

$$
r_{1}(x)=\left\{\begin{array}{ll}
1-\frac{x^{2}}{2}-i x\left(1-\frac{x^{2}}{4}\right)^{1 / 2} ; & |x| \leqslant 2, \\
1-\frac{x^{2}}{2}+|x|\left(\frac{x^{2}}{4}-1\right)^{1 / 2} ; & |x| \geqslant 2,
\end{array} \quad x=\frac{\omega h}{2}\right.
$$

We can explain this result as follows: when the wavelength is large compared to $h$, the Fourier transform of $v_{j}$ is a left-travelling wave, and when the wavelength is small, it is an evanescent wave.

The proof of this lemma (see [6, p. 175]) uses limit absorption techniques: one first introduces the perturbed absorbing problem, then passes to the limit by using the Fourier transform.

Using "cut-off" and "smoothing" techniques, we apply this lemma to $u_{j}$, and obtain the transparent boundary condition:

Proposition 3.2 (TRANSPARENT Boundary CONDITION). The solution $\left(u_{j}\right)$ of the space-discretized problem satisfies the relation

$$
\forall T \in] 0,+\infty[, \forall t \in] 0, T\left[: u_{-1}(t)=\int_{-\infty}^{+\infty} \int_{0}^{T} r_{1}^{-1}(\omega h) u_{0}(t) e^{i \omega(t-\tau)} d \tau d \omega,\right.
$$

where $r_{1}$ is given by (1.10).

Fundamental Remark. We have obtained an expression of $u_{-1}$ in terms of $u_{0}$. We could also have obtained an expression of $u_{0}$ in terms of $u_{-1}$ :

$$
u_{0}(t)=\int_{-\infty}^{+\infty} \int_{0}^{T} r_{1}(\omega h) u_{-1}(t) e^{i \omega(t-\tau)} d \tau d \omega
$$

We shall now deduce the approximate boundary conditions.

3.1.2. Investigation of Local Boundary Conditions. The method consists in approximating the kernel $r_{1}^{-1}(\omega h)$ of relation (3.11) (or the local kernel $r_{1}$ of relation (3.12)) when the frequency $\omega$ is small. We use Taylor or Padé expansions. Recalling that $i \omega$ corresponds to $d / d t$, we obtain the derivatives of $u_{0}$ or $\left(u_{0}-u_{-1}\right) / h$.

When $\omega h$ is small, we have

$$
\begin{aligned}
& r_{1}^{-1}(\omega h)=1-\frac{\omega^{2} h^{2}}{2}+i \omega h\left(1-\frac{\omega^{2} h^{2}}{4}\right)^{1 / 2} ; \\
& r_{1}(\omega h)=1-\frac{\omega^{2} h^{2}}{2}-i \omega h\left(1-\frac{\omega^{2} h^{2}}{4}\right)^{1 / 2} .
\end{aligned}
$$

Using the first Taylor expansion, we get

$$
\begin{aligned}
& \frac{d u_{0}}{d t}+\frac{u_{0}-u_{-1}}{h}=0 \\
& \frac{d u_{-1}}{d t}+\frac{u_{0}-u_{-1}}{h}=0
\end{aligned}
$$


(3.13) is a natural discretization of the transparent boundary condition

$$
\partial u / \partial t+\partial u / \partial x=0
$$

The next Taylor expansion gives

$$
\begin{gathered}
\frac{d u_{0}}{d t}+\frac{u_{0}-u_{-1}}{h}+\frac{h}{2} \frac{d^{2} u_{0}}{d t^{2}}=0 \\
\frac{d u_{-1}}{d t}+\frac{u_{0}-u_{-1}}{h}-\frac{h}{2} \frac{d^{2} u_{-1}}{d t}=0
\end{gathered}
$$

And the first Padé expansion gives the reciprocity formulae

$$
\begin{aligned}
& \frac{d u_{0}}{d t}+\frac{u_{0}-u_{-1}}{h}-\frac{h}{2} \frac{d}{d t}\left(\frac{u_{0}-u_{-1}}{h}\right)=0, \\
& \frac{d u_{-1}}{d t}+\frac{u_{0}-u_{-1}}{h}+\frac{h}{2} \frac{d}{d t}\left(\frac{u_{0}-u_{-1}}{h}\right)=0 .
\end{aligned}
$$

Obviously we are able to obtain boundary condition up to any order.

We will say that the boundary condition is of order $l$, if the expansion is of order $l$.

3.1.3. Stability of the Boundary Conditions. We study the stability with energy techniques:

We denote by $E_{h}(t)$ the energy in the half-axis $x<0$, at time $t . E_{h}$ is given by

$$
E_{h}=\frac{1}{2} \sum_{j \leqslant-1} h\left(\frac{d u_{j}}{d t}\right)^{2}+\frac{1}{2} \sum_{j \leqslant-1} h\left(\frac{u_{j}-u_{j-1}}{h}\right)^{2} .
$$

Then, the following energy estimates hold:

$$
\frac{d}{d t} E_{h}=\frac{d u_{-1}}{d t} \cdot \frac{u_{0}-u_{-1}}{h}
$$

and

$$
\frac{d}{d t} E_{h} \leqslant \frac{d u_{0}}{d t} \cdot \frac{u_{0}-u_{-1}}{h}
$$

We can now deduce

Proposition 3.3. All the approximate boundary conditions written in 3.1 .2 are stable: The energy at any time is bounded by the energy at the initial time. Moreover the boundary conditions (3.13) and (3.13bis) are absorbing.

From these stable approximate boundary conditions, we can derive stable boundary conditions for the discretized problem.

3.2. Approximate Boundary Conditions for the Discretized Problem. We determine these conditions by discretization of the conditions (3.13)-(3.15bis). We use a centered divided difference for the time-derivatives, in order to obtain-whenever it is true-the stability by energy methods.

Indeed the energy identity is

$$
\left(E_{n+1}-E_{n}\right) / 2 \Delta t-D_{0}^{t}\left(u_{-1}^{n}\right) D_{-}^{x}\left(u_{0}^{n}\right)=0 .
$$

We have seen that the discretization of (3.13) leads to an ill-posed boundary problem. On the other hand, the equality (3.19) shows that, from the condition (3.14bis), we obtain a stable boundary condition. 
We can prove also that the boundary conditions of second order, deduced from (3.14) and (3.14bis), are stable, but the one deduced from (3.15) is not.

Hence, the method does not determine systematically stable boundary conditions. But, as far as we know, a systematic method to obtain such boundary conditions does not yet exist (see, for example, [4]).

Therefore, we can sum up our results:

Proposition 3.4. The two following boundary conditions hold:

(1) $D_{0}^{t}\left(u_{-1}^{n}\right)+D_{-}^{x}\left(u_{0}^{n}\right)=0$,

(it is absorbing).

$$
\text { (2) } D_{0}^{t}\left(u_{0}^{n}\right)+D_{-}^{x}\left(u_{0}^{n}\right)+\frac{\Delta x}{2} \frac{D_{+}^{t}-D_{-}^{t}}{\Delta t}\left(u_{0}^{n}\right)=0
$$

(it is stable, and transparent when $\Delta t=\Delta x$ ).

Notice that the boundary condition of second order (3.21) is close to the one written by B. Engquist and A. Majda (see [4]), but here the stability is given by energy estimates.

Example. The initial velocity is equal to 0 . The initial state is the following:

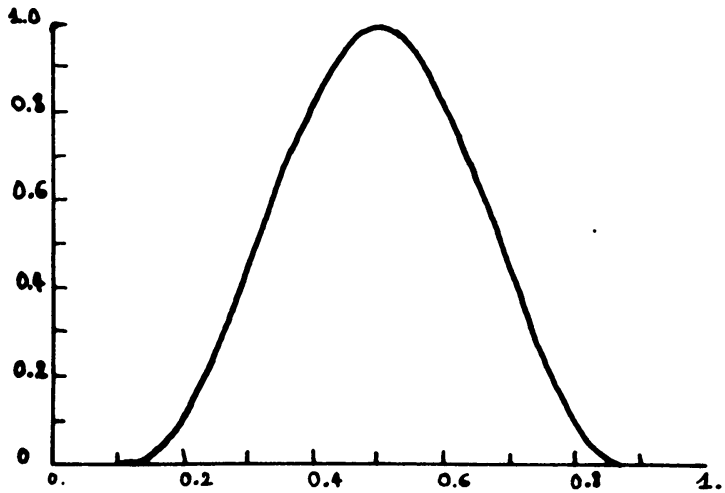

Figure 2

We impose the boundary conditions at $x=0$ and $x=1$.

We take $\gamma=0.1, \Delta x=1 / 64$. Solutions are drawn at time $T=1$, the exact solution being zero.

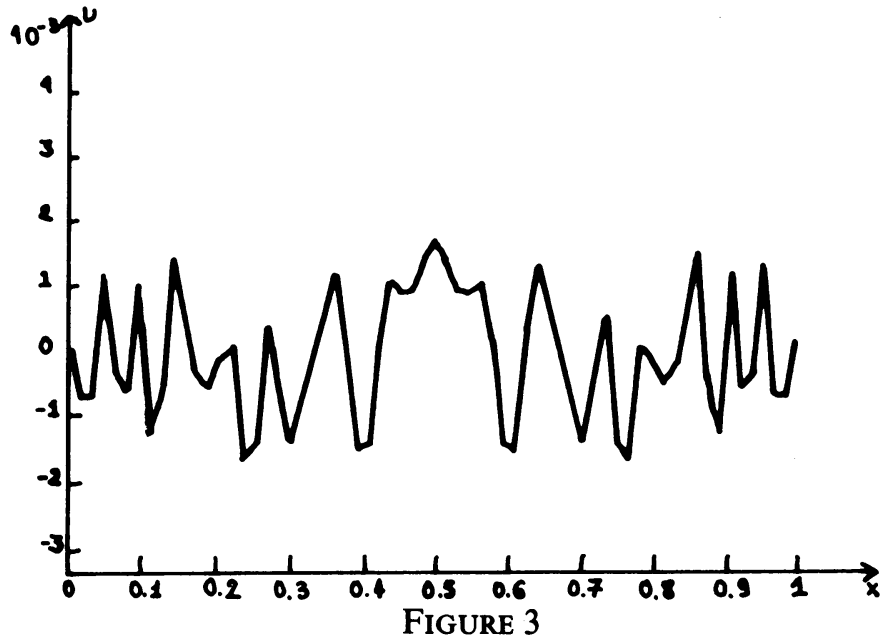

Boundary condition (1.20) 


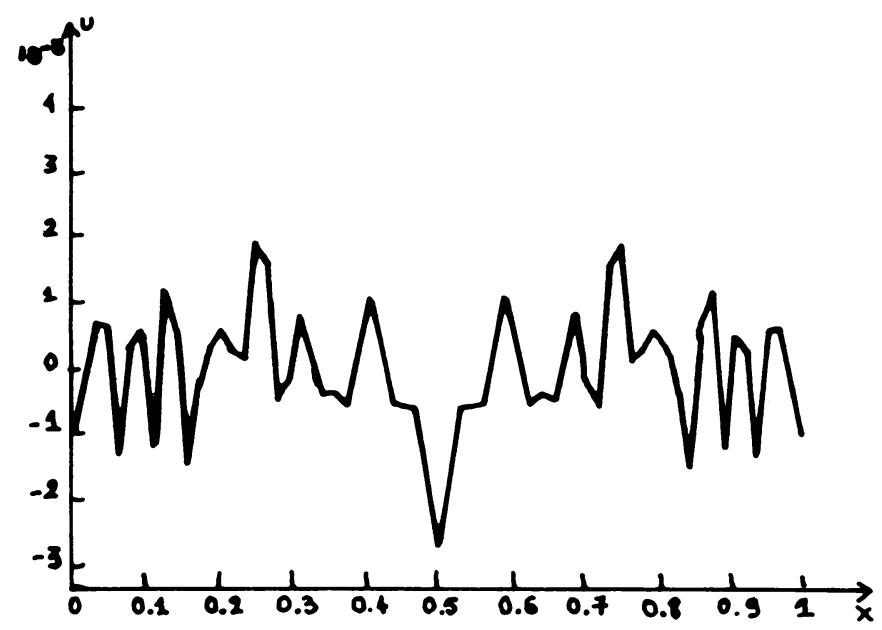

FIGURE 4

Boundary condition of Engquist and Majda

Conclusion. For other discretization schemes, the sketch of our method will be the following:

(1) We obtain stable boundary conditions on the space-discretized problem at the point $j=0$ with Fourier techniques.

(2) We discretize in time with centered divided differences at the point $j=-1$.

3.3. Error Estimates. We shall see that, under regularity conditions for the solution, we can obtain error estimates for the solution of the problem in the artificial domain: the accuracy of this method being the order of boundary conditions.

We denote by $u_{j}^{n}$ the solution of the problem on $\mathbf{R}$, and $v_{j}^{n}$ the solution of the problem on the half-line $x<0$, with one of the boundary conditions (3.20), (3.21), which we denote by $B C_{\Delta t}$. Let

$$
w_{j}^{n}=v_{j}^{n}-u_{j}^{n} ; \quad j \leqslant 0 .
$$

$w_{j}^{n}$ is the reflected part of $u_{j}^{n}$ by the boundary condition. $\left(w_{j}^{n}\right)_{j \leqslant 0}$ is a solution of the initial boundary problem on $]-\infty, 0[$, with initial values identically zero and a boundary condition at $j=0$

$$
B C\left(w_{0}, w_{-1}\right)=-B C\left(u_{0}, u_{-1}\right) \text {. }
$$

Let $u_{j}(t)=u_{j}^{n} ; v_{j}(t)=v_{j}^{n} ; w_{j}(t)=w_{j}^{n}$ on $]\left(n-\frac{1}{2}\right) \Delta t,\left(n+\frac{1}{2}\right)[$.

On the half-line $j \leqslant 0, u_{j}$ satisfies the relation

$$
\frac{u_{j}(t-\Delta t)-2 u_{j}(t)+u_{j}(t+\Delta t)}{\Delta t^{2}}-\frac{u_{j+1}(t)-2 u_{j}(t)+u_{j-1}(t)}{\Delta x^{2}}=0 .
$$

The same equation holds for $v_{j}$ and $w_{j}$.

This remark leads us to the space-discretized problem.

3.3.1. Error Estimates for the Space-Discretized Problem. We keep the same notations: $u_{j}$ denotes the solution of the problem

$$
\frac{d^{2} u_{j}}{d t^{2}}-\frac{u_{j+1}-2 u_{j}+u_{j-1}}{h^{2}}=0 ; \quad j \in \mathbf{Z},
$$

with initial values in $K=[l, m], m<0$. 
$v_{j}$ denotes the solution of the same problem on the half-line $j<0$ with one of the boundary conditions written in 3.1 .2 , and $w_{j}=v_{j}-u_{j}$ is the part of $u_{j}$ reflected by the boundary condition $B C$.

As in Proposition 3.1, we can obtain the expressions of the Fourier-transforms of $u_{j}$ and $w_{j}$ where $r_{1}$ is defined in (3.10). (See Figure 5.)

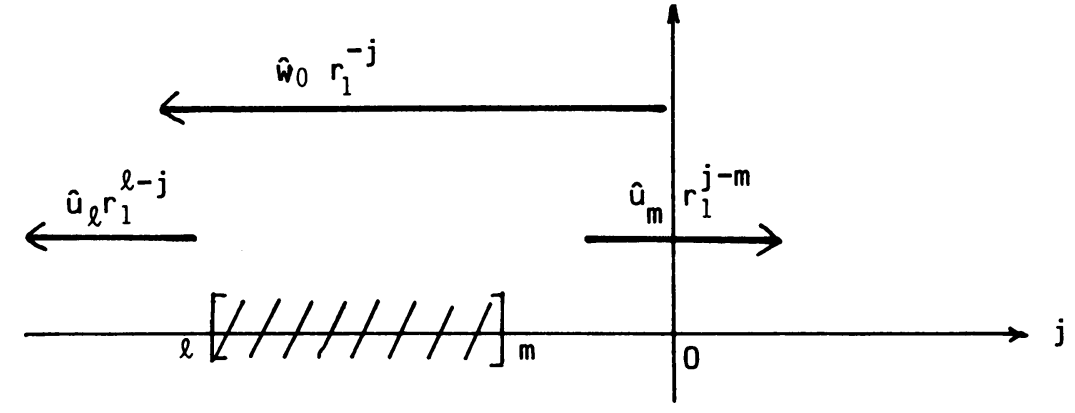

FIGURE 5

The reflection coefficient $R$ is defined with the Fourier transforms of $u_{j}$ and $w_{j}$ by

$$
\hat{w}_{0}(\omega)=R(\omega h) \hat{u}_{0}(\omega) \text {. }
$$

We can estimate $R$ by

LEMMA 3.2. The reflection coefficient satisfies the estimate

$$
|R(\omega h)| \leqslant|\omega h|^{l}
$$

where $l$ is the order of the boundary condition.

This estimate is deduced from the explicit form of the reflection coefficient for each boundary condition.

In order to obtain error estimates, we must get estimates on the time-derivatives and discrete space-derivatives of $w_{h}$.

LEMMA 3.3. If $l$ is the order of the boundary condition, we have the following estimates:

$$
\begin{gathered}
\forall T \in] 0,+\infty\left[; \forall j \leqslant 0: \int_{0}^{T}\left(\frac{d w_{j}}{d t}\right)^{2} d t \leqslant C h^{2 l} \sum_{k=0}^{l} \int_{0}^{T}\left(\frac{d^{k+1} u_{m-j}}{d t^{k+1}}\right)^{2} d t\right. \\
\int_{0}^{T}\left(\frac{w_{j}-w_{j-1}}{h}\right)^{2} d t \leqslant C h^{2 l} \sum_{k=0}^{l} \int_{0}^{T}\left(\frac{d^{k}}{d t^{k}}\left(\frac{u_{m-j}-u_{m-j+1}}{h}\right)\right)^{2} .
\end{gathered}
$$

We only give here the proof of (3.26), in the case where the boundary condition is (3.13):

$$
\frac{d u_{0}}{d t}+\frac{u_{0}-u_{-1}}{h}=0 ; \quad l=1 .
$$

The other cases can be treated by a similar method.

*First, we prove the result for $j=0$ :

From the explicit forms of $\hat{w}_{j}$ and $\hat{u}_{j}$, given in Figure 5, we deduce a relation between $\hat{w}_{0}$ and $\hat{u}_{m}: \hat{w}_{0}=r_{1}^{-m} \hat{u}_{m}$, which gives

$$
\frac{{\widehat{d w_{0}}}_{d t}}{d t} h\left(\frac{r_{1}^{-m} R(\omega h)}{i \omega h}\right) \frac{\widehat{d}^{2} u_{m}}{d t^{2}} .
$$


Under the assumption that $d^{2} u_{m} / d t^{2}$ lies in $L^{2}(] 0,+\infty[)$, and using the Parseval inequality, we get

$$
\int_{0}^{+\infty}\left(\frac{d w_{0}}{d t}\right)^{2} \leqslant C h \int_{0}^{+\infty}\left(\frac{d^{2} u_{m}}{d t^{2}}\right)^{2} d t .
$$

Here, $d^{2} u_{m} / d t^{2}$ is not in $L^{2}(] 0,+\infty[)$, but in $L^{2}(] 0, T[)$ for each $T>0$. It is easy to see that this problem may be reduced to ]0, $T$ [. Indeed the map $d u_{m} / d t \mapsto w_{0}$ is causal, that is the value of $w_{0}$ at time $T$ depends only on the values of $d u_{m} / d t$ at time $t \leqslant T$. Then, by truncation and regularization, we can use the Parseval inequality to get

$$
\int_{0}^{T}\left(\frac{d w_{0}}{d t}\right)^{2} \leqslant C h \int_{0}^{T}\left(\left(\frac{d u_{m}}{d t}\right)^{2}+\left(\frac{d^{2} u_{m}}{d t^{2}}\right)^{2}\right) d t
$$

*Now, for each $j \leqslant 0$, we have the following relation between $\hat{w}_{j}$ and $\hat{u}_{m-j}$ :

$$
\hat{w}_{j}=R r_{1}^{-m} \hat{u}_{m-j},
$$

which is independent of $j$. Since it is causal for $j=0$, it is too for every $j$. The result (3.26) follows as in the first step.

We are now able to obtain, with the usual regularity assumptions on the initial value,

THEOREM 3.1 (ERROR ESTIMATES). Let $l$ be the order of the boundary condition.

Assuming that the initial state is in $H^{l+1}(] 0,+\infty[)$ and the initial velocity is in $H^{\prime}(] 0,+\infty[)$, we have the following estimate on the energy of the reflected part:

$$
\forall T \in] 0,+\infty\left[: \frac{1}{T} \int_{0}^{T} E_{h}(w h)(t) d t \leqslant C h^{2 l},\right.
$$

where $C$ is a constant, depending only on the initial values.

Practically, we introduce two artificial boundaries with the same boundary condition. This leads to a difficulty: we then have to deal with two reflections at the same time:

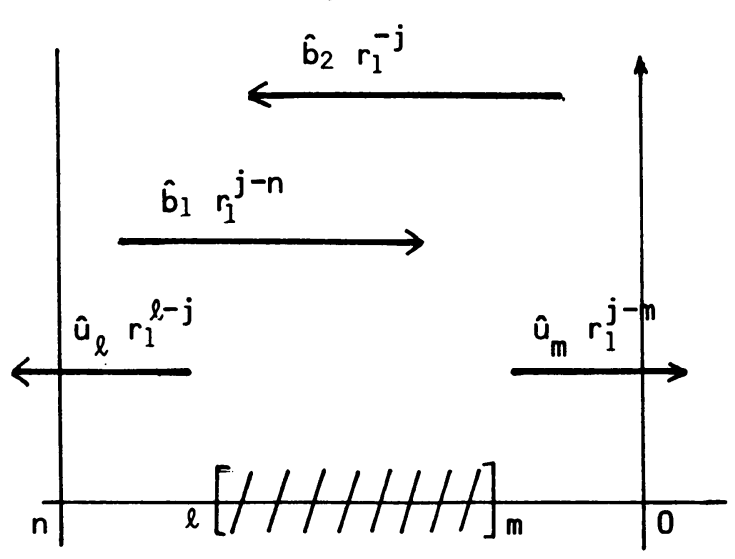

Figure 6

$$
\hat{w}_{j}=\hat{b}_{1} r_{1}^{j-m}+\hat{b}_{2} r_{1}^{-j} \text {. }
$$


We have to use, instead of a reflection coefficient, a reflection matrix

$$
\left(\begin{array}{c}
\hat{w}_{j-n} \\
\hat{w}_{j}
\end{array}\right)=K^{(j)}\left(\begin{array}{c}
\hat{u}_{m+j} \\
\hat{u}_{l-j}
\end{array}\right)
$$

The matrix $K^{(j)}$ depends on $r_{1}$ and $R$. It has a singularity at the critical frequency, i.e., $|\omega h|=2$, where $r_{1}=-1$ and $R=-1$. Therefore, we obtain less accurate estimates for $K^{(j)}$ than for $R$. On the other hand, if we consider the mean-value of $w_{h}$ on two space-steps

$$
\tilde{w}_{j}=\frac{w_{j}+w_{j+1}}{2},
$$

we get for $\frac{1}{2}\left(K^{(j)}+r_{1} K^{(j+1)}\right)$ the same accurary.

LEMMA 3.4. If $l$ is the order of the boundary condition at $j=0$ and $j=n$, the following estimates on the reflection matrix $K^{(j)}$ hold:

$$
\begin{gathered}
\left\|K^{(j)}\right\| \leqslant C(j+1)|\omega h|^{l} ; \quad n / 2 \leqslant j \leqslant 0, \\
\| \frac{1}{2}\left(K^{(j)}+r_{1} K^{(j+1)} \| \leqslant C|\omega h|^{l} ; \quad n / 2 \leqslant j \leqslant 0,\right.
\end{gathered}
$$

where $C$ does not depend on $\omega, j, h$.

Then, the same arguments as in Lemma 3.3 and Theorem 3.1 give

THEOREM 3.2 (ERROR ESTIMATES ON A BOUNDED DOMAIN). If $w_{h}=\left(w_{j}\right)$ is the reflected part of $u_{h}=\left(u_{j}\right)$ by two artificial boundaries, where the same boundary condition of order $l$ is imposed, under the regularity assumptions of Theorem 3.1, we have

$$
\begin{gathered}
\forall T \in] 0,+\infty\left[; \quad \frac{1}{T} \int_{0}^{T} E_{h}\left(w_{h}\right)(t) d t \leqslant C h^{2(l-1)},\right. \\
\forall T \in] 0,+\infty\left[; \quad \int_{0}^{T} E_{h}\left(\tilde{w}_{h}\right)(t) d t \leqslant C h^{2 l},\right.
\end{gathered}
$$

where $\tilde{w}_{h}$ is the mean-value of $w_{h}$ on two space-steps, given by (3.30).

We remark that, in (3.33), the order of the estimate we get is one less than the one in (3.34). In particular, for the first order boundary conditions (3.13) and (3.13bis), we only have

$$
\frac{1}{T} \int_{0}^{T} E_{h}\left(w_{h}\right)(t) d t \leqslant C
$$

In order to improve this result, we derive an $L^{\infty}$ estimate on $w_{h}$.

THEOREM 3.3 ( $L^{\infty}$ ESTIMATES). Under the assumptions of Theorem 3.2, we have

$$
\forall T \in] 0,+\infty\left[: E_{h}\left(w_{h}\right)(t) \leqslant C h^{2 l-1} \int_{0}^{T} E_{h}\left(\frac{d^{l} u_{h}}{d t^{l}}\right)(t) d t\right.
$$

We are now able to obtain error estimates for the discrete problem. 
3.3.2. Error Estimates for the Discretized Problem. Suppose that $u_{j}$ is in $L^{2}(] 0,+\infty[)$. We can introduce the Fourier transform of $u_{j}$. It satisfies the equation

$$
\hat{u}_{j+1}-2\left(1-\frac{1}{2}\left(\left|\frac{\sin \frac{\omega \Delta t}{2}}{\frac{\omega \Delta t}{2}}\right|^{\omega}\right)^{2} h^{2} \hat{u}_{j}+\hat{u}_{j-1}=0\right.
$$

Here $\hat{u}_{j}$ can be written as

$$
\hat{u}_{j}=\hat{a}_{1} r_{1, \Delta t}^{j}+\hat{a}_{2} r_{1, \Delta t}^{-j},
$$

where $r_{1}, \Delta t$ is given in terms of $r_{1}$ (introduced in (1.10)) by

$$
r_{1, \Delta t}(\omega)=r_{1}\left(\left|\frac{\sin \frac{\omega \Delta t}{2}}{\frac{\omega \Delta t}{2}}\right| \omega\right)
$$

When $\Delta t$ tends to zero, the boundary condition $B C_{\Delta t}$ tends to a boundary condition $B C$, which is of the same order. The reflection coefficient $R_{\Delta t}$ of $B C_{\Delta t}$ is given in terms of the reflection coefficient $R$ of $B C$ by

$$
R_{\Delta t}(\omega)=R\left(\left|\frac{\sin \frac{\omega \Delta t}{2}}{\frac{\omega \Delta t}{2}}\right| \omega\right)
$$

It is periodic, and when the order of $B C_{\Delta t}$ is $l$, we have $\left|R_{\Delta t}(\omega)\right| \leqslant|\omega \Delta x|^{l}$.

Therefore we have the following estimate:

Assuming that the solution $u_{j}^{n}$ of the initial value problem is in $l^{2}(\mathbf{N})$, the following error estimate for the energy of the reflected part $w_{j}$ at $j=0$ holds:

$$
\frac{1}{N} \sum_{0}^{N} E_{n} \leqslant C h^{2 l}
$$

where $l$ is the order of the boundary condition, and $C$ is a constant depending only on the initial values.

We shall now see how our method can be used to construct stable boundary conditions for finite element schemes.

4. Application of the Method to the Finite Element $e^{1}$. We consider here an approximation of the wave equation with cubic finite elements: the real-axis is divided into segments $\left[x_{i}, x_{i+1}\right]$ of length $h$. The approximation space is the space of functions $\mathcal{C}^{1}$, which are polynomial of degree 3 on each segment. The degrees of freedom are the values of the function and its derivative at the point $x_{j}$.

Let

$$
u_{j}=\left(\begin{array}{c}
u\left(x_{j}\right) \\
h u^{\prime}\left(x_{j}\right)
\end{array}\right)
$$


The partially discretized problem can be written as follows (cf. [13]).

$$
M h^{2} \frac{d^{2} U_{j}}{d t^{2}}=A_{-1} U_{j-1}+A_{0} U_{j}+A_{1} U_{j+1} .
$$

$M, A_{-1}, A_{0}, A_{1}$ are $2 \times 2$ matrices, $M$ is the mass matrix.

4.1. Dispersion Relation. Formally, we can apply the Fourier transform to Eq. (4.2). On the two half-lines, where the initial values vanish, $\hat{U}_{j}$ satisfies the equation

$$
A_{-1} \hat{U}_{j-1}+\left(A_{0}+\omega^{2} h^{2} M\right) \hat{U}_{j}+A_{1} \hat{U}_{j+1}=0 \text {. }
$$

The solutions of (4.3) have the form $\hat{U}_{j}=r^{j} \hat{U}$, where $r$ and $U$ are related by

$$
\left(A_{-1}+r\left(A_{0}+\omega^{2} h^{2} M\right)+r^{2} A_{1}\right) \hat{U}=0 .
$$

(4.4) is the dispersion relation.

We deduce in particular the form of the harmonic wave solutions of (4.2):

LEMMA 4.1. The harmonic wave solutions of (4.2) can have any of the following forms:

(1) The travelling waves:

$$
\hat{U}_{j}=e^{i(\omega t+k j h)} \hat{U}, \quad e^{i k h} \text { and } \hat{U} \text { being related by }(4.4)
$$

(2) The evanescent waves:

$$
\hat{U}_{j}=e^{i \omega t+\nu j h} \hat{U}, \quad e^{\nu h} \text { and } \hat{U} \text { being related by (4.4). }
$$

4.2. Transparent Boundary Condition. By the same arguments as in Lemma 3.1, we deduce from the dispersion relation an expression of $\hat{U}$; on the right-hand side of the compact support of the initial condition

$$
\hat{U}_{j}(\omega h)=r_{1}^{j}(\omega h) \hat{U}^{(1)}(\omega h)+r_{2}^{j}(\omega h) \hat{U}^{(2)}(\omega h),
$$

where $\left(r_{1}, \hat{U}^{(1)}\right)$ and $\left(r_{2}, \hat{U}^{(2)}\right)$ are related by (4.4).

So, $\hat{U}_{j}$ is the sum of two waves which are either travelling or evanescent.

Proposition 4.1. The transparent boundary condition for the partially discretized problem has the following form

$$
\hat{U}_{0}=\left(\hat{U}^{(1)}, \hat{U}^{(2)}\right)\left(\begin{array}{cc}
r_{1} & 0 \\
0 & r_{2}
\end{array}\right)\left(\hat{U}^{(1)}, \hat{U}^{(2)}\right)^{-1} \hat{U}_{-1} .
$$

From now on, the mass matrix is assumed to be the identity.

4.3. Discretization of the Continuous Transparent Boundary Condition by Finite Elements. We have seen that the transparent boundary condition for the continuous problem is $\partial u / \partial t+\partial u / \partial x=0$.

By discretization, we get the discrete boundary condition

$$
U_{-1}=\left(\begin{array}{cc}
1 & -1 / 3 \\
0 & 3
\end{array}\right) U_{0}+h\left(\begin{array}{cc}
2 / 3 & 0 \\
2 & 0
\end{array}\right) \frac{d U_{-1}}{d t}
$$

This boundary condition is clearly stable.

4.4. Approximate Boundary Conditions. In order to obtain approximate boundary conditions, we expand $r_{1}, r_{2}, \hat{U}^{(1)}, \hat{U}^{(2)}$ of relation (4.8), when the wavelength is large compared to $h$. 
For example, with Taylor expansion of order 1, we get

$$
U_{-1}=\left(\begin{array}{cc}
1 & -1 / 3-\frac{\sqrt{2}}{6} \\
0 & 3+2 \sqrt{2}
\end{array}\right) U_{0}+h\left(\begin{array}{cc}
\frac{2}{3}-\frac{\sqrt{2}}{6} & \frac{2 \sqrt{2-1}}{36} \\
2+2 \sqrt{2} & \frac{\sqrt{2+1}}{2}
\end{array}\right) \frac{d U_{-1}}{d t}
$$

It can be proved, by energy techniques, that this boundary condition is stable.

4.5. Comparison Between (4.9) and (4.10) in Terms of Reflection. We investigate here the reflection of an evanescent wave by the two boundary conditions, when the wavelength is large compared with $h$.

An evanescent wave is reflected as the sum of an evanescent wave and a travelling wave.

In the first case, i.e., the boundary condition (4.9), the amplitude of the reflected travelling wave increases as $1 / \omega h$ when $\omega h$ tends to 0 ; in the second case, i.e., the boundary condition (4.10) calculated by our method, the amplitude of the reflected travelling wave decreases as $\omega h$ : the evanescent wave is absorbed.

In order to obtain a stable boundary condition for the discretized problem, we discretize the term $d U_{-1} / d t$ of (4.10) with a centered divided difference, and we get finally:

THEOREM 4.1. The following boundary condition of first order holds:

$$
U_{-1}^{n}=\left(\begin{array}{cc}
1 & -\frac{1}{3}-\frac{\sqrt{2}}{6} \\
0 & 3+2 \sqrt{2}
\end{array}\right) U_{0}^{n}+h\left(\begin{array}{cc}
\frac{2}{3}-\frac{\sqrt{2}}{6} & \frac{2 \sqrt{2-1}}{36} \\
2+2 \sqrt{2} & \frac{\sqrt{2+1}}{2}
\end{array}\right) \frac{U_{-1}^{n+1}-U_{-1}^{n-1}}{\Delta t}
$$

It is absorbing.

Obviously, we must calculate boundary conditions of order larger than 1. This calculation is more difficult, and we will use the computer; see [6].

Conclusion. We have, for two schemes of space-discretization (finite differences and finite elements), approximate boundary conditions which are stable. For the finite difference scheme, the boundary condition is close to the one available in earlier papers (see for example [4] and [8]).

But, and this is new in the field, as far as we know, we obtained error estimates for the reflected part. And for the finite element discretization scheme, the results obtained with our boundary conditions, particularly in the case of nonsmooth initial values (evanescent waves), are better than the ones using variational formulation.

Of course, one can apply our method to any discretization scheme of the wave equation; see [6].

Centre de Mathématiques Appliquées

Ecole Polytéchnique

91128 Palaiseau Cedex, France 
1. P. Betres \& O. C. ZienkiewiCZ, "Diffraction and refraction of surfaces waves using finite and infinite elements," Internat. J. Numer. Methods Engrg., v. 11, 1977, pp. 1271-1290.

2. R. W. Clayton \& B. ENGQuist, "Absorbing boundary conditions for the numerical solution of wave propagation problems,” SSA Bull., v. 6, 1977, pp. 1529-1540.

3. R. W. CLAYTON \& B. ENGQUist, "Absorbing boundary conditions for wave-equation migration," Geophysics, v. 45, 1980, pp. 895-904.

4. B. ENGQuist \& A. MAJDA, "Absorbing boundary conditions for numerical simulation of waves," Math. Comp., v. 31, 1977, pp. 629-651.

5. B. ENGQuist \& A. MAJDA, "Boundary conditions for acoustic and elastic wave calculations," Comm. Pure Appl. Math., v. 32, 1979, p. 313.

6. L. HALPERN, Etude de Conditiones aux Limites Absorbantes pour des Schémas Numériques Relatifs à des Equations Hyperboliques Linéaires, Thèse de 3éme cycle, Université Paris VI, Septembre 1980.

7. H. O. KreIss, "Initial boundary value problems for hyperbolic systems," Comm. Pure Appl. Math., v. 23, 1970, pp. 277-298.

8. E. L. LindmanN, “"Free-space” boundary conditions for the time dependent wave equation," $J$. Comput. Phys., v. 18, 1975, pp. 66-78.

9. A. MAJdA \& S. OSHER, "Reflection of singularities at the boundary," Comm. Pure Appl. Math., v. 28,1975 , pp. $479-491$.

10. L. NiRENBERG, Lectures on Linear Partial Differential Equations, CBMS Regional Conf. Ser. in Math., no. 17, Amer. Math. Soc., Providence, R. I., 1973; reprinted 1979.

11. A. C. REYNOLDS, "Boundary conditions for the numerical solution of wave propagation problems," Geophysics, v. 43, 1978, pp. 1099-1110.

12. W. D. SMITH, "A non reflecting plane boundary for wave propagation problems," J. Comput. Phys., v. 15,1974 , pp. $492-503$.

13. G. Strang \& G. J. Fix, Analysis of the Finite Element Method, Prentice-Hall, Englewood Cliffs, N. J., 1973.

14. M. E. TAYLOR, "Reflecting of singularities of solutions to systems of differential equations," Comm. Pure Appl. Math., v. 28, 1975, pp. 457-478. 